\title{
RUÍDO E SEUS IMPACTOS NOS HOSPITAIS BRASILEIROS: UMA REVISÃO DE LITERATURA
}

\section{Noise and its impact on Brazilian hospitals: a literature review}

\author{
Walderes Aparecida Filus ${ }^{(1)}$, Liane Fuhr Pivatto(2), Francisca Pinheiro Fontoura( ${ }^{(3)}$, \\ Marilu Rita Villa Koga( ${ }^{(4)}$, Evelyn Joice Albizu(5), Vânia Muniz Néquer Soares( ${ }^{(6)}$, \\ Adriana Bender Moreira de Lacerda( ${ }^{(7)}$, Cláudia Giglio de Oliveira Gonçalves ${ }^{(8)}$
}

\begin{abstract}
RESUMO
O aumento dos níveis de ruído nos hospitais, atribuído a diferentes fatores, dentre eles a grande incorporação de tecnologias, a concentração e o fluxo de pessoas e a não observância do silêncio pela própria equipe de trabalho e usuários do serviço, pode impactar negativamente no processo de trabalho e na saúde de profissionais e usuários. Este estudo teve como objetivo realizar revisão de literatura nacional sobre ruído em serviços hospitalares no Brasil, analisando aspectos referentes à produção científica, aos métodos de mensuração, aos níveis de ruído presentes nos hospitais, e às conclusões e estratégias propostas nos estudos analisados. O nível de ruído nos hospitais brasileiros está acima dos padrões recomendados acarretando impactos nesses serviços. Existe um relativo acúmulo de conhecimento sobre o tema no Brasil. Para ampliação desse conhecimento foram recomendados estudos de intervenção para mitigação do excesso de ruídos nos hospitalais. O controle do ruído nos hospitais deve ser considerado como uma das prioridades para melhoria de sua ambiência.
\end{abstract}

DESCRITORES: Medição de Ruído; Ruído Ocupacional; Unidades Hospitalares

\section{INTRODUÇÃO}

Os hospitais têm se transformado em locais ruidosos, devido a diferentes fatores, dentre eles, a grande incorporação de tecnologias ${ }^{1}$. Nas unidades de terapia intensiva (UTIs) são numerosas as fontes

(1) Departamento de Enfermagem da Universidade Tuiuti do Paraná - UTP, Curitiba, Paraná, Brasil.

(2) Universidade Tuiuti do Paraná - UTP, Curitiba, Paraná, Brasil.

(3) Universidade Tuiuti do Paraná - UTP, Curitiba, Paraná, Brasil.

(4) Universidade Tuiuti do Paraná - UTP, Curitiba, Paraná, Brasil.

(5) Universidade Tuiuti do Paraná - UTP, Curitiba, Paraná, Brasil.

(6) Universidade Tuiuti do Paraná, UTP, Curitiba, Paraná, Brasil.

(7) Universidade Tuiuti do Paraná - UTP, Curitiba, Paraná, Brasil.

(8) Programas de Mestrado e Doutorado em Distúrbios da Comunicação da Universidade Tuiuti do Paraná - UTP, Curitiba, Paraná, Brasil.

Conflito de interesses: inexistente geradoras de ruído, como aspiradores, monitores, ventiladores mecânicos, computadores, impressoras e saídas de ar comprimido ${ }^{2-8}$.

O ruído destas tecnologias e os provenientes do ar condicionado, sons de instrumentos de trabalho, concentração e trânsito de pessoas, transporte de pacientes e reformas de estruturas físicas, estão entre os fatores de riscos ocupacionais que podem comprometer a saúde do trabalhador de enfermagem ${ }^{9-14}$.

O aumento do ruído em hospitais relaciona-se ainda com a não observância do silêncio pela própria equipe de trabalho e pelos usuários do serviço. No entanto, o silêncio se faz necessário para o bom funcionamento da rotina hospitalar, incluindo a recuperação dos pacientes ${ }^{15,16}$.

A Agência Nacional de Vigilância Sanitária do Ministério da Saúde (ANVISA/MS) ${ }^{17}$ reconhece o risco ocupacional no ambiente hospitalar, destacando os níveis elevados de ruído nas centrais de compressão de ar e geração de vácuo, nas oficinas de manutenção, nas marcenarias e nas lavanderias 18, pelo grande número de máquinas existentes nesses locais. A ANVISA menciona que 
nas UTIs, os modernos equipamentos com alarmes sonoros, emitem ruídos de menor intensidade, porém, causam incômodos.

As equipes de saúde realizam várias tarefas no ambiente hospitalar, que exigem atenção constante para que não ocorram erros no processo de trabalho. Um estudo realizado em ambiente hospitalar identificou que o processo de preparo e administração de medicamentos ocorrendo em ambiente ruidoso, dificulta a concentração dos profissionais e os induz a erros ${ }^{19}$.

Com relação aos níveis de ruído, a Organização Mundial de Saúde ${ }^{20}$ recomenda que, nos ambientes hospitalares, não ultrapasse $30 \mathrm{~dB}(\mathrm{~A})$. A Associação Brasileira de Normas Técnicas (ABNT) ${ }^{21}$ normatiza níveis entre 35 e $55 \mathrm{~dB}(\mathrm{~A})$ para hospitais.

A Norma Brasileira- NBR n. ${ }^{0}$ 10152/8722 registrada no Instituto Nacional de Metrologia, Normalização e Qualidade Industrial (INMETRO), estabelece para os hospitais valores de níveis sonoros de $35 \mathrm{~dB}(\mathrm{~A})$ para conforto, e $45 \mathrm{~dB}(\mathrm{~A})$ como valor aceitável para apartamentos, enfermarias, berçários e centros cirúrgicos; laboratórios e áreas para uso do público, $40 \mathrm{~dB}(\mathrm{~A})$ para conforto e $50 \mathrm{~dB}(\mathrm{~A})$ como nível aceitável para a finalidade; serviços, 45 e $55 \mathrm{~dB}(\mathrm{~A})$.

Em relação à saúde do trabalhador, o Capítulo V da Consolidação das Leis do Trabalho (CLT), por meio da Portaria n. ${ }^{0} 3214 / 78$, estabelece, pela Norma Regulamentadora n. ${ }^{0} 15$ (NR-15) ${ }^{23}$, limites de tolerância ou de exposição ocupacional ao ruído, sendo para ruído contínuo ou intermitente o nível-critério de $85 \mathrm{~dB}(\mathrm{~A})$ para um período de oito horas de exposição. Nos locais de trabalho onde são executadas atividades que requerem atenção e uso intelectual, é recomendado pela Norma Regulamentadora n. ${ }^{0} 17$ (NR-17) ${ }^{24}$ seguir as condições de conforto acústico estabelecidas pela Norma Brasileira (NBR) n. ${ }^{0} 10152^{22}$, o nível aceitável é de até $65 \mathrm{~dB}(\mathrm{~A})$.

Níveis de ruído elevados podem causar distúrbios comportamentais, resultando em respostas fisiológicas ao estresse em pacientes hospitalizados. A intensidade da pressão sonora em $65 \mathrm{~dB}(\mathrm{~A})$ pode afetar o hipotálamo e a hipófise, elevando os níveis de secreção de adrenalina, noradrenalina e corticosteróides, bem como causar aumento da pressão arterial e alterações no ritmo cardíaco e vasoconstrição periférica ${ }^{3,25}$. Há relatos na literatura sobre a relação entre os níveis de pressão sonora elevados e a perda auditiva, o estresse, as alterações psicológicas e do sono, podendo causar lapsos de memória e um maior esforço mental na realização das tarefas, expondo o grupo a riscos de acidentes e erros na execução do seu trabalho ${ }^{2,26}$.
Devido ao risco potencial que o ruído representa para pacientes e equipes de saúde nos hospitais, indica-se que a sua mensuração seja realizada e que os níveis sonoros sejam identificados, o que favorecerá a implantação de mudanças de controle efetivo e redução de ruídos ${ }^{27,28}$.

Nessa perspectiva, o presente trabalho objetivou realizar revisão de literatura sobre o ruído em serviços hospitalares no Brasil, analisando os diferentes aspectos referentes à produção científica relacionada com o tema, os métodos que foram utilizados para mensuração do ruído nos hospitais, os resultados obtidos, bem como as conclusões e as estratégias propostas nos estudo analisados.

\section{MÉTODOS}

A revisão de literatura foi realizada a partir das bases de dados on-line MEDLINE, LILACS e SCIELO, utilizando-se as palavras-chave: ruído and hospital and ambiente, ruído hospitalar e pressão sonora no hospital. Os critérios de inclusão foram os estudos quantitativos sobre ruído hospitalar no Brasil, publicados na íntegra, em língua portuguesa, no período de janeiro de 2000 a março de 2011. Foram excluídos os estudos não disponibilizados na íntegra nas bases de dados pesquisadas, os que não se encontravam no limite temporal em questão e os que não mensuravam os níveis de ruído. Após a análise dos critérios de inclusão, foram identificados 17 estudos, considerados relevantes para o presente trabalho. Deste total, 14 são artigos publicados e 3 são dissertações.

Os estudos foram enumerados de 1 a 17 , e para a coleta e síntese dos dados, um protocolo foi elaborado com as variáveis relevantes para análise e discussão, tais como o ano de publicação, formação profissional dos autores das publicações, metodologia utilizada para a mensuração do ruído, normas científicas e as principais conclusões e recomendações encontradas. As análises dos estudos incluíram ainda as descrições dos procedimentos, os parâmetros para a avaliação do ruído, os resultados obtidos quanto aos níveis de ruídos nos diferentes ambientes hospitalares e as estratégias de ações propostas para a redução do ruído.

\section{REVISÃO DA LITERATURA}

$\mathrm{Na}$ Tabela 1, apresenta-se a distribuição dos artigos segundo $\mathrm{o}$ ano de publicação, área $\mathrm{e}$ formação profissional dos autores. Verificou-se que nos últimos cinco anos houve aumento do número de pesquisas sobre ruído hospitalar no Brasil. No período de 2000-2005, identificaram-se 6 
publicações, enquanto que no período de 2006-2011 há quase o dobro, com 11 publicações. O maior número de publicações ocorreu em periódicos de Enfermagem $(47,1 \%)$ e nos periódicos da área médica $(41,2 \%)$. Estudos publicados em periódicos na área de fonoaudiologia e administração representaram $5,9 \%$ do total analisado. Em relação à formação profissional dos autores, verificou-se que $35,3 \%$ das publicações foram desenvolvidas por grupos de pesquisadores de diferentes formações.

Tabela 1 - Distribuição dos artigos segundo o ano de publicação, área e profissão dos autores ( $\mathrm{N}=17)$

\begin{tabular}{lcc}
\hline Ano & N.o & $\%$ \\
\hline $2006-2011$ & 11 & 65 \\
$2000-2005$ & 6 & 35 \\
\hline Área de publicação dos Periódicos & & \\
Enfermagem & 8 & 47,1 \\
Área médica & 7 & 41,2 \\
Fonoaudiologia & 1 & 5,9 \\
Administração & 1 & 5,9 \\
\hline Formação profissional dos autores & & \\
Multiprofissional (médicos,enfermeiros, engenheiros, & 6 & 35,3 \\
fonouadiólogos,etc) & 6 & 17,6 \\
Médico & 3 & 17,6 \\
Enfermeiro & 3 & 11,8 \\
Fonoaudiólogo & 2 & 5,9 \\
Engenheiro & 1 & 5,9 \\
Arquiteto & 1 & 5,9 \\
Não consta & 1 & \\
\hline
\end{tabular}

A ampliação da produção de estudos no período de 2006-2011 ocorreu, possivelmente, como consequência do interesse de diferentes profissionais da saúde, relacionado à percepção da mudança do perfil de ruído nos serviços hospitalares, como também pelo avanço da engenharia sobre o ruído e o conhecimento sobre seus efeitos para a audição e a saúde em geral. Observou-se que o maior número de publicações ocorreu em periódicos de Enfermagem. Isso pode ser justificado pela ampliação do papel do Enfermeiro junto à área de Saúde do Trabalhador, cuja atribuição de qualificação requer constante busca de desenvolvimento e aprofundamento de conhecimentos. Além disso, este profissional planeja ações pautadas no processo de trabalho, que são relevantes para a execução de programas de conscientização sobre os efeitos do ruído no ambiente hospitalar ${ }^{29}$.

Observou-se ainda que a maior parte das publicações foi de equipes multiprofissionais. Isto indica a interação dos profissionais de diversas áreas, configurando uma relação recíproca entre as múltiplas intervenções técnicas e a interação de agentes $^{30}$. Um dos exemplos de trabalho coletivo são as várias áreas profissionais, gerando uma rede de relações entre pessoas, poderes, saberes, afetos, e desejos, nas quais é possível identificar os processos grupais ${ }^{31}$. Constitui uma estratégia capaz de melhorar a qualidade de vida da população, porque busca a integralidade dos cuidados de atenção à saúde das pessoas. 
$\mathrm{Na}$ Tabela 2, observa-se a distribuição dos artigos segundo local do estudo e área do hospital avaliada. $\mathrm{Na}$ análise dos dados observou-se que a maioria dos estudos foi realizada em hospitais públicos de ensino $(52,94 \%)$, seguidos de hospitais públicos gerais $(29,41 \%)$. Com referência à área hospitalar pesquisada, revelou-se a predominância das UTIs, uma vez que $58,8 \%$ dos estudos foram realizados nessas áreas. Evidenciou-se ainda, a predominância de estudos realizados em UTI neonatais e de Cuidados Intermediários Pediátricos/ UCI-pediátricas, correspondentes a $52,9 \%$ do total analisado.

Tabela 2 - Distribuição dos artigos segundo local do estudo e área do hospital ( $N=17)$

\begin{tabular}{lcc}
\hline Variável & N.o & $\%$ \\
\hline Local do estudo & & 52,94 \\
Hospital de Ensino Público & 9 & 29,41 \\
Hospital Público Geral & 5 & 11,76 \\
Hospital Privado & 2 & 5,88 \\
Não descrito & 1 & \\
\hline Área avaliada do Hospital & & 29,4 \\
UTI Neo (Unidade de Terapia Intensiva Neonatal) & 5 & 23,5 \\
UTI (Unidade de Terapia Intensiva) & 4 & 11,8 \\
Diversas áreas & 2 & 11,8 \\
UCIN (Unidade de Cuidados Intensivos Neonatais ) & 2 & 11,8 \\
Lavanderia & 2 & 5,9 \\
UTI Pediátrica & 1 & 5,9 \\
CC (centro cirúrgico) & 1 & \\
\hline
\end{tabular}

Os hospitais de ensino ou universitários são as principaisinstituiçõesquecomplementam, emtermos práticos, a formação prática dos profissionais de saúde. Além disso, são voltados para atendimentos de média e alta complexidade, e contam com maior número de equipamentos e circulação de pessoas. Referente às áreas dos hospitais, as UTIs são locais particularmente afetados pelo ruído. Nelas, são empregados muitos equipamentos dotados de alarmes acústicos, essenciais para alertar médicos e enfermeiros sobre alterações das condições clínicas sofridas pelos pacientes ou sobre o mau funcionamento dos próprios aparelhos ${ }^{2}$. Assim, esses ambientes que deveriam ser silenciosos e tranquilos, tornam-se ruidosos e estressantes. Para o paciente, pode ainda aumentar a ansiedade e a percepção dolorosa, diminuir o sono e prolongar o tempo de convalescença ${ }^{32,33}$.

Nas UTIs neonatais, segundo alguns pesquisadores $^{34}$, o ambiente superestimulante presente nesses setores pode comprometer o processo de desenvolvimento e crescimento, especialmente dos prematuros que têm receptores sensoriais extremamente sensíveis ao ambiente. Há indícios de que o sistema auditivo do prematuro, pela sua própria imaturidade geral, é provavelmente mais suscetível às lesões que podem ser causadas por esse ambiente, em razão da combinação do ruído com o uso de medicações ototóxicas, que aumentam ainda mais o risco. Devido a isso, a partir da década de 70 em alguns países - no Brasil, somente na década de 90 - tiveram início os estudos direcionados à questão do ruído em ambientes neonatais, especificamente em incubadoras. Outros pesquisadores $^{28}$ observam que o ambiente tecnológico da Unidade de Cuidados Intensivos oferece benefícios em termos de equilíbrio biológico, no entanto, é física e psicologicamente agressivo, no qual, o nível de ruído constitui uma das interações nocivas.

Os dados apresentados na Tabela 3 referem-se ao protocolo de avaliação do ruído utilizado nos artigos nacionais. Nos estudos revisados, em aproximadamente $3 / 4$ deles $(76,5 \%)$, o equipamento utilizado na avaliação do ruído foi o medidor de nível de pressão sonora, e em $23,5 \%$ dos estudos constatou-se opção pelo dosímetro de ruído como medida para obtenção do nível equivalente. 
Tabela 3 - Dados dos protocolos de avaliação do ruído utilizado nos estudos ( $N=17)$

\begin{tabular}{lcc}
\hline Variável & N.o & $\%$ \\
\hline Equipamentos utilizados para avaliação & & \\
Medidor de nível de pressão sonora & 13 & 76,5 \\
Dosímetro & 4 & 23,5 \\
\hline Normas utilizadas para comparação & & \\
Nacional & 8 & 47,1 \\
Nacional e Internacional & 6 & 35,3 \\
Não consta & 2 & 11,8 \\
Internacional & 1 & 5,9 \\
\hline
\end{tabular}

Outro fator analisado foi o tempo de mensuração registrado nos estudos, demonstrado na Figura 1. Observa-se que cada pesquisador utilizou uma sistemática diferente, de acordo com as necessidades e requisitos do local e do tema de estudo, e/ ou equipamento de medição utilizado, podendo-se notar a diversidade de tempos de mensuração, de intervalos entre as mensurações e de duração das medições. Verificou-se que a maioria das medições foi realizada nos três turnos( manhã, tarde e noite). Em três estudos realizaram-se as medições nos horários considerados de pico, ou seja, no período da manhã. O tempo de medição variou de 27,30 e 60 segundos a 5,10 e 15 minutos. Em quatro estudos foi realizada medição de forma ininterrupta (24 h). No que se refere às normas utilizadas para a análise dos dados dos estudos, constata-se que $47,1 \%$, optaram por comparar aos valores de níveis de ruído recomendados em normas nacionais, enquanto que $35,3 \%$ dos pesquisadores optaram por utilizar simultaneamente as normas nacionais e internacionais.

\begin{tabular}{|c|c|c|}
\hline $\begin{array}{l}\text { Tempo de mensuração em } \\
\text { minutos/aproximados por estudo (E no )* }\end{array}$ & $\begin{array}{l}\text { Intervalo entre as } \\
\text { medições }\end{array}$ & $\begin{array}{l}\text { Duração das medições } \\
\text { em dias e/ou horas }\end{array}$ \\
\hline 6000 (E.1) & $27 s$ & 8,12 ou $24 \mathrm{~h}$ \\
\hline 8640 (E.2) & 0 & 6 dias $-24 h$ \\
\hline 2400 (E.3) & $1 \mathrm{~min}$ & 7 dias $-24 h$ \\
\hline 5160 (E.4) & 0 & 7 dias $-12 h$ \\
\hline 5040 (E.5) & 0 & 14 dias $-12 h$ \\
\hline 360 (E.6) & $\mathrm{N} / \mathrm{C}$ & $1 \mathrm{dia}-6 \mathrm{~h}$ \\
\hline $10080 \quad(\mathrm{E} .7)$ & $5 \mathrm{seg}$ & 7 dias $-24 h$ \\
\hline Instantânea (E.8) & $10 \mathrm{~min}$ & 3 dias $-3 h$ \\
\hline 69 medidas/registro (E.9) & $30 s$ & 14 dias $-12 \mathrm{~h}$ \\
\hline 3480 (E.10) & $15 \mathrm{~min}$ & $\mathrm{~N} / \mathrm{C}$ \\
\hline (E.11) & $\mathrm{N} / \mathrm{C}$ & $1 \mathrm{dia}-4 \mathrm{~h}$ \\
\hline (E.12) & $\mathrm{N} / \mathrm{C}$ & 13 dias -2 a $4 \mathrm{~h}$ \\
\hline $51840($ E.13) & 0 & 9 dias- $24 h$ \\
\hline $33 \min (\mathrm{E} .14)$ & $1 \mathrm{~min}$ & 11 dias $-6 h$ \\
\hline $45 \min (\mathrm{E} .15)$ & $5 \min$ & 3 dias- $3 h$ \\
\hline 5760 registros $(\mathrm{E} .16)$ & 0 & 4 dias- $24 \mathrm{~h}$ \\
\hline (E.17) & $\mathrm{N} / \mathrm{C}$ & 2 dias $-12 h$ \\
\hline
\end{tabular}

* Numero do ESTUDO

**Pontuais: ruído de impacto

N/C: Não consta a informação

Figura 1 - Distribuição do tempo de mensuração, intervalos entre medições e duração das medições em cada estudo 
A NBR $10151^{21}$ estabelece que o tempo de medição deve ser escolhido de forma a permitir a caracterização do ruído. A medição pode envolver uma única amostra ou uma sequência delas. Isso justifica a grande diversidade de padrões utilizados nos estudos. As normas nacionais incluem a Norma Regulamentadora NR 15 da Portaria 3214/78 do Ministério do Trabalho e Emprego quando o risco for ocupacional, ou seja, com níveis de ruído acima de $85 \mathrm{~dB}(\mathrm{~A})$ para 8 horas de exposição ${ }^{23}$, e a NBR 10152/1987 da ABNT - Associação Brasileira de Normas Técnicas, recomenda 35 a $45 \mathrm{~dB}(\mathrm{~A})$ para conforto acústico (ABNT, 1996). As normas internacionais utilizadas são as recomendações da OMS -Organização Mundial da Saúde, cujo nível equivalente para diferentes ambientes hospitalares não deve ser superior a $40 \mathrm{~dB}(\mathrm{~A})$ para o período diurno e $35 \mathrm{~dB}(\mathrm{~A})$ para o período noturno. Outra norma internacional, do Committee to Establish Recommended Standards for Newborn ICU
Design, estabelece que o ruído habitual da UCIN - Unidade de Cuidados Intensivos Neonatal e UTIN - Unidade de Terapia Intensiva Neonatal, não deve exceder o nível equivalente de $50 \mathrm{~dB}(\mathrm{~A})$. A Academia Americana de Neonatologia, baseada na Environmental Protection Agency/74 dos Estados Unidos, recomenda um nível de pressão sonora não superior a $45 \mathrm{~dB}(\mathrm{~A})$ diurno e $35 \mathrm{~dB}(\mathrm{~A})$ noturno. Pode-se verificar que os níveis recomendados estão muito próximos, tanto nacionais quanto internacionais, havendo um consenso nos valores indicados.

Na Figura 2, apresentam-se os níveis de ruído encontrados por área de estudo, quando utilizados os equipamentos medidores de nível de pressão sonora, comumente conhecidos como decibelímetro, assim como, o dosímetro de ruído. $\mathrm{Na}$ mensuração dos níveis de pressão sonora, verificou-se que, a média mínima foi de $50.1 \mathrm{~dB}(\mathrm{~A})$ e máxima de $96.0 \mathrm{~dB}(\mathrm{~A})$.

\begin{tabular}{|c|c|c|c|c|}
\hline Área Hospitalar & Máxima dB(A) & Mínima dB(A) & Média em Leq dB(A) & $\begin{array}{l}\text { Observação } \\
\text { dB(A) }\end{array}$ \\
\hline \multicolumn{5}{|c|}{ MEDIDOR DE NPS } \\
\hline$(\mathrm{E} .1)^{\star} \mathrm{UTI}$ geral & 108.4 & 40 & $\begin{array}{l}65.36 \\
\text { Diurno- } 65.23 \\
\text { Noite - } 63.89\end{array}$ & $\begin{array}{l}\text { A média Leq } \\
\text { variou de: } 62.9 \\
\text { a } 69.3\end{array}$ \\
\hline$(\mathrm{E} .2)^{*}$ UTI Pediátrica & 120.0 & & 60.0 a 70.0 & nível basal \\
\hline $\begin{array}{l}(\mathrm{E} .3)^{\star} \text { Diversas áreas do } \\
\text { hospital }\end{array}$ & 58.0 & 101.0 & $\begin{array}{l}63.7 \\
\text { Recepção PS }-64.2 \\
\text { Sala de gesso }-60.6 \\
\text { UTI Neonatal }-61.4 \\
\text { UTI - } 62.7 \\
\text { Centro Cirúrgico }-59.1 \\
\text { Centro de mat. estéril. }-66.0 \\
\text { Farmácia }-63.3 \\
\text { Pediatria }-60.0 \\
\text { Cozinha }-62.9 \\
\text { Lavanderia }-71.5\end{array}$ & \\
\hline$(\mathrm{E} .6)^{\star} \mathrm{UTI}$ & $\begin{array}{l}1-80.4 \\
2-82.4 \\
\text { Coronariana- } 73.3\end{array}$ & $\begin{array}{l}1-80.4 \\
2-82.4 \\
\text { Coron. - } 73.3\end{array}$ & $\begin{array}{l}1-64.1 \\
2-64.0 \\
\text { Coronariana }-58.9\end{array}$ & \\
\hline$(\mathrm{E} .7)^{\star} \mathrm{UTI}$ & & & $\begin{array}{l}\text { Diurno }-60.86 \pm 4.90 \\
\text { Noturno }-55.60 \pm 5.98 \\
\text { Dias úteis }-58.21 \pm 5.93 \\
\text { Final de sem- } 56.83 \pm 5.90 \\
\text { Troca de plantão: } \\
\text { Diurna }-61.35 \pm 5.08 \\
\text { Noturna }-62.31 \pm 4.70 \\
\text { Visita: mat.- } 60,50 \pm 4.59 \\
\text { Vespertina- } 62.04 \pm 4.48 \\
\text { Noturna }-60.05 \pm \mathbf{4 . 2 7}\end{array}$ & \\
\hline$(\mathrm{E} .7)^{\star}$ Centro Cirúrgico & 96,0 vapor ligado & & $\begin{array}{l}\text { Recepção- } 73.6 \text { a } 83.6 \\
\text { Corredor- } 68.2 \text { a } 85.0 \\
\text { Sala cirúrgica- } 66.4 \text { a } 79.2 \\
\text { Expurgo- } 79.9 \text { a } 83.8 \\
\text { Preparo material- } 68.1 \text { a } 83.6 \\
\text { Esterilização- } 69.6 \text { a } 96.0\end{array}$ & 86.5 na $C M$ \\
\hline$(\text { E.9 })^{\star}$ Incubadoras - (impacto) & $116 \mathrm{~dB}(\mathrm{C})$ & $76,1 \mathrm{~dB}(\mathrm{C})$ & & \\
\hline
\end{tabular}




\begin{tabular}{|c|c|c|c|c|}
\hline Área Hospitalar & Máxima dB(A) & Mínima dB(A) & Média em Leq dB(A) & $\begin{array}{l}\text { Observação } \\
\text { dB(A) }\end{array}$ \\
\hline $\begin{array}{l}(\text { E.10)*Estabelecimentos de } \\
\text { Saúde }\end{array}$ & $\begin{array}{l}\text { 94.9 Abastecimento } \\
\text { de } \mathrm{O}^{2}\end{array}$ & $\begin{array}{l}\text { 45.9 Higiêne do } \\
\text { paciente }\end{array}$ & ------- & \\
\hline$($ E.11)* PAIR em Lavanderia & & & $\begin{array}{l}\text { Todo o Hospital }-\mathbf{7 5 . 0} \\
\text { Lavanderia Matutino }-\mathbf{9 1 . 0} \\
\text { Lavand. Vespertino }-\mathbf{9 0 . 0} \\
\text { Cozinha matutino }-\mathbf{8 1 . 0} \\
\text { Cozinha vespertino }-\mathbf{8 0 . 0} \\
\text { Marcenaria matutino }-\mathbf{8 0 . 0} \\
\text { Marcenaria vespertino }-\mathbf{7 5 . 0} \\
\text { Corredor dos ambulatórios } \\
\text { matutino }-\mathbf{7 5 . 0} \\
\text { Corredor dos ambulatórios } \\
\text { vespertino }-\mathbf{5 5 . 0}\end{array}$ & \\
\hline$(\mathrm{E} .12)^{\star} \mathrm{UTI}$ neonatal & $\begin{array}{l}\text { Manhã - } 80.4 \text { (Lavagem } \\
\text { mãos) } \\
\text { Noite } 78.1 \text { (Troca de } \\
\text { equipamentos) }\end{array}$ & & $\begin{array}{l}\text { Manhã } \mathbf{8 4 . 5} \\
\text { Tarde } \mathbf{7 6 . 6} \\
\text { Noite } \mathbf{7 6 . 6}\end{array}$ & \\
\hline$(E .14)^{\star}$ UTI neonatal & & & 50.1 a 62.0 & \\
\hline$(\text { E.15) })^{\star}$ Lavanderia & $\begin{array}{l}\text { Manhã - } \mathbf{9 8 . 0} \\
\text { Tarde }-\mathbf{9 7 . 0} \\
\text { Noite }-\mathbf{1 0 1 . 0}\end{array}$ & $\begin{array}{l}\text { Manhã } \mathbf{- 8 1 . 0} \\
\text { Tarde }-\mathbf{7 6 . 0} \\
\text { Noite } \mathbf{- 7 0 . 0} \\
\end{array}$ & ------- & \\
\hline$(\mathrm{E} .17)^{\star} \mathrm{UTI}$ & $\begin{array}{l}\text { Diurno }-\mathbf{8 9 . 3} \\
\text { Matutino }-\mathbf{8 9 . 3} \\
\text { Vespertino }-\mathbf{8 3 . 4}\end{array}$ & $\begin{array}{l}\text { Diurno }-55.6 \\
\text { Matutino }-61.4 \\
\text { Mínima }-\mathbf{5 5 . 6}\end{array}$ & $\begin{array}{l}\text { Diurno }-\mathbf{7 1 . 0} \\
\text { Matutino }-\mathbf{7 1 . 6} \\
\text { Vespertino }-\mathbf{7 0 . 4}\end{array}$ & \\
\hline \multicolumn{5}{|c|}{ DOSÍMETRO } \\
\hline$(E .4)^{*}$ UCIN Neonatal & & & 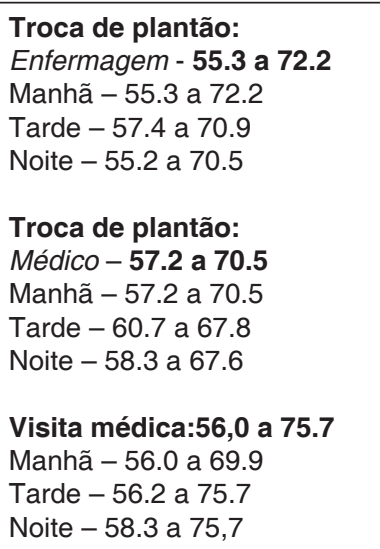 & $\begin{array}{l}\text { Valor limite } \\
\text { para UCIN: } 50 \\
\mathrm{~dB}(\mathrm{~A})\end{array}$ \\
\hline$(E .5)^{*}$ UCIN Neonatal & $\begin{array}{l}\text { Sexta - 54,5 a } 82,2 \\
\text { Sábado - 54,2 a } 86,0 \\
\text { Domingo - 52,6 a } 72,6 \\
\text { Segunda }-52,7 \text { a } 76,2 \\
\text { Terça - 53,0 a } 71,6 \\
\text { Quarta - } 51,8 \text { a } 71,6 \\
\text { Quinta - 52,8 a } 71,6\end{array}$ & $\begin{array}{l}\text { Sexta- 52,0 } \\
\text { Sabado- 51,9 } \\
\text { Dom- } \mathbf{5 1 , 7} \\
\text { Seg- 51,9 } \\
\text { Terça- 50,7 } \\
\text { Quart- } 51,2 \\
\text { Quint- } \mathbf{5 2 , 1}\end{array}$ & $\begin{array}{l}\text { Sexta - 61,1 } \\
\text { Sábado - 59,5 } \\
\text { Domingo - 60,9 } \\
\text { Segunda - 60,1 } \\
\text { Terça - 62,3 } \\
\text { Quarta - 59,6 } \\
\text { Quinta - 61,6 }\end{array}$ & \\
\hline$(E .13)^{\star} \mathrm{UTI}$ neonatal & $\begin{array}{l}\text { Cuidados intens. - } 114.1 \\
\text { Cuidados Interm. - } 90.2 \\
\text { Isolamento - } 100.8 \\
\text { Corredor - } 104.9\end{array}$ & & $\begin{array}{l}\text { cuidados intensivos - } 64.8 \\
\text { Cuidados Interm. }-62.1 \\
\text { Isolamento }-63.8 \\
\text { Corredor }-61.9\end{array}$ & \\
\hline$($ E.16)*UTIN neonatal & $\begin{array}{l}96.8 \text { a } 121.0 \mathrm{~dB}(\mathrm{C}) \\
90.8 \text { a } 100.3 \mathrm{~dB}(\mathrm{C}) \\
98.9 \text { a } 123.4 \mathrm{~dB}(\mathrm{C}) \\
103,4 \text { a107.6 dB(C) }\end{array}$ & & $\begin{array}{l}5^{\text {a }} \text { feira }-62,2 \\
6^{a} \text { feira }-61.3 \\
\text { Sábado }-66,0 \\
\text { Domingo- } 66.6\end{array}$ & $\begin{array}{l}\text { Noturno } \\
123,4 \mathrm{~dB}(\mathrm{C}) \\
\text { Matutino } \\
\text { 103.4dB(C) } \\
\text { Vespertino } \\
90.8 \mathrm{~dB}(\mathrm{C})\end{array}$ \\
\hline
\end{tabular}

$\mathrm{N}^{*}$ Número do Estudo

UCIN Unidade de Cuidados Intensivo Neonatal

Figura 2 - Demonstrativo das áreas hospitalares estudadas e dos níveis de ruído encontrados utilizando o medidor de nível de pressão sonora e dosímetro de ruído 
Quanto aos níveis de ruído detectados nos diferentes estudos, observa-se que estão acima dos valores recomendados pelas normas nacionais e internacionais tanto para a saúde ocupacional como para conforto acústico. Verifica-se que há níveis de pressão sonora os quais podem causar perda auditiva induzida pelo ruído, uma vez que se encontram acima do limite de tolerância indicado na Norma Regulamentadora NR 15, sendo um risco ocupacional aos profissionais das lavanderias hospitalares, bem como aos usuários de UTIs gerais e neonatais e de outras áreas hospitalares. A análise dos estudos demonstra ainda, que os níveis de ruído que estão abaixo do limite de tolerância que consta da NR 15, devem ser comparados aos limites recomendados pelas normas da ABNT para conforto acústico determinado pela NBR $10152^{22}$.

Quanto à especificação dos equipamentos de medição, o método e os procedimentos para avaliação são indicados na NBR $10151^{21}$, de junho de 2000. A norma recomenda que o medidor de nível de pressão sonora ou o sistema de medição atenda às especificações da norma IEC 60651 para o tipo 0 , tipo 1 ou tipo 2 e que o equipamento de avaliação possua recursos para medição de nível de pressão equivalente ponderado em "A" (Leq).

O nível equivalente (Leq) é definido como sendo o nível sonoro estacionário, que se ocorresse durante o intervalo de registro, geraria a mesma energia sonora produzida pelos eventos sonoros registrados. Alguns medidores fornecem uma leitura direta do Leq em intervalos de tempo de escolha do pesquisador ${ }^{35}$. Alguns modelos de medidores de nível de pressão sonora possuem este recurso, assim como alguns modelos de dosímetros de ruído. Portanto, estes dois tipos de equipamentos podem ser utilizados nas avaliações desde que atendam ao recomendado pelas normas regulamentadoras. Por definição ${ }^{35}$, o dosímetro de ruído foi projetado para ser um aparelho de monitoramento de uso pessoal, permitindo a medição da dose e outras grandezas, caracterizando a exposição do trabalhador ao ruído, mas atualmente estes aparelhos podem fornecer muitas informações relativas à avaliação do ruído em ambientes de trabalho, atendendo às diferentes normas e legislação, além da avaliação ambiental considerando o conforto acústico.

Finalizando essa revisão da literatura, inclui-se a síntese das conclusões e recomendações relacionadas ao ruído hospitalar nos estudos, conforme a Tabela 4. Observando-se diferentes desfechos ou conclusões, predomina o nível de ruído acima do recomendado nos hospitais brasileiros, com $42,85 \%$ dos estudos, a necessidade de conscientização dos profissionais em $17,85 \%$ dos estudos, e que o ruído é mais acentuado no período diurno em $14,28 \%$.

Tabela 4 - Síntese das conclusões e recomendações relacionados ao ruído hospitalar segundo os estudos analisados

\begin{tabular}{lcc}
\hline Variáveis & $\mathbf{N .}^{{ }^{0}}$ & $\%$ \\
\hline Sintese das conclusões dos estudos & 12 & 42,85 \\
Níveis de ruído acima do recomendado & 5 & 17,85 \\
Conscientização dos profissionais & 4 & 14,28 \\
Ruído acentuado no período diurno & 3 & 10,71 \\
Provoca agravos à saúde do trabalhador & 2 & 7,14 \\
Não há risco ocupacional & 1 & 3,57 \\
Profissionais conhecem a origem do ruído & 1 & 3,57 \\
Ruído considerado intenso e moderado pelos profissionais & 28 & 100 \\
\hline Total & & \\
\hline Sintese das recomendações dos estudos & 8 & 38.09 \\
Educação sobre os efeitos do ruído & 4 & 19.04 \\
Monitoramento do ruído & 3 & 14.28 \\
Adequação sonora dos equipamentos & 3 & 14.28 \\
Ajustes arquitetônicos & 3 & 14.28 \\
Envolvimento dos gestores & 21 & 100 \\
\hline Total
\end{tabular}

Obs.: Alguns estudos citam mais de uma conclusão e recomendação 
Em relação às recomendações apresentadas pelos autores, mais de $1 / 3(39,09 \%)$ das pesquisas citaram a educação sobre os efeitos do ruído. O monitoramento do ruído foi referido em 19,04\% dos estudos. Outras três sugestões citadas foram a adequação sonora dos equipamentos, os ajustes arquitetônicos e o envolvimento dos gestores, respectivamente em $14,28 \%$ dos estudos.

Enfim, os estudos referem que os estabelecimentos de saúde deveriam contar com espaço físico com condições básicas de conforto ambiental, dentre eles o conforto acústico, o que beneficiaria o paciente e a todos os envolvidos no processo ${ }^{10}$. Como recomendações para diminuir o ruído no ambiente, a grande maioria dos estudos citou a educação e conscientização sobre os efeitos do ruído, a necessidade de adequações do ambiente físico visando ao maior conforto sonoro e a manutenção preventiva dos equipamentos e a aquisição de equipamentos com emissão de níveis de ruído reduzidos. Tais fatores possibilitarão à equipe hospitalar atuar de modo mais efetivo na redução da poluição sonora, beneficiando assim, a função laborativa dos profissionais e a recuperação dos pacientes, reduzindo seu impacto nos hospitais brasileiros.

\section{CONSIDERAÇÕES FINAIS}

Esta revisão evidenciou que existe um relativo acúmulo de conhecimento sobre o ruído hospitalar no Brasil, suas fontes geradoras e seus efeitos na saúde. Recomendam-se, portanto, estudos de intervenção, com a experimentação e relatos de experiências sobre estratégias para a mitigação do excesso de ruídos nos diferentes ambientes hospitalares. Assim, amplia-se o conhecimento e contribui-se com resultados positivos na prática. Foi possível observar, pelos estudos desta revisão, que o controle do ruído nos hospitais deve ser considerado uma das prioridades para melhoria da sua ambiência. Dessa forma, considera-se importante o investimento em estudos que mostrem as diferentes formas de exposição ao ruído no ambiente hospitalar a que estão sujeitos tanto profissionais como usuários, e o impacto na saúde dessa população, visando a sua correção e à implementação de medidas preventivas.

\section{CONCLUSÃO}

O maior número de publicações ocorreu no período de 2006 a 2011, em periódicos de enfermagem e desenvolvidos por grupos de pesquisadores multiprofissionais, sendo a maior parte em hospitais públicos de ensino, predominantemente em UTIs. O equipamento mais usado foi o medidor de nível de pressão sonora, e cada pesquisador desenvolveu uma sistemática diferente para as medições, assim como o tempo de mensuração. A maior parte das medições foi realizada nos três períodos. A maioria dos dados foi comparada com as normas nacionais. Em todos os estudos, o ruído nos hospitais brasileiros estava acima dos padrões recomendados.

\begin{abstract}
The increase in noise levels at hospitals could be attributed to many factors as incorporation of new technologies, concentration and flow of people and non-compliance of silence by the workers and patients. Noise provokes negatively impact the work process and people's health. This study aimed a review the national literature on noise in hospital services in Brazil, by the analysis of the follow aspects: scientific research, measuring methods, noise levels in hospitals, and the findings and proposed strategies in studies. The noise level in Brazilian hospitals is above the recommended levels. There is an increasing knowledgement of this subject in Brazil. In order to expand this knowledge, intervention studies were recommended to eliminate the excessive noise into the hospitals. The noise control in hospitals should be considered as a priority to improve their surroundings.
\end{abstract}

KEYWORDS: Noise Measurement; Occupational Noise; Hospital Units 


\section{REFERÊNCIAS}

1. Otenio MH, Cremer E, Claro EMT. Intensidade de ruído em hospital de 222 leitos na 18a Regional de Saúde - PR. Rev. Bras. Otorrinolaringol. 2007; 73(2):245-50.

2. Pereira RP, Toledo RN, Amaral JLG, Guilherme A. Qualificação e quantificação da exposição sonora ambiental em uma unidade de terapia intensiva geral. Rev. bras. Otorrinolaringol. 2003 Nov.dez; 69(6):766-71.

3. Carvalho WB, Pedreira MLG, Aguiar MAL. Nível de ruídos em uma unidade de cuidados intensivos pediátricos. J. pediatr. 2005 Nov/dez;81(6):495-8.

4. Kakehashi TY, Pinheiro EM, Pizzarro G, Guilherme A. Nível de ruído em unidade de terapia intensiva neonatal. Acta Paul. Enferm. 2007; 20:404-9.

5. Sampaio Neto RA, Mesquita FOS, Junior MDSPJ, Ramos FMDA, Junior MAVC. Ruídos na Unidade de terapia Intensiva: quantificação e percepção dos profissionais de saúde. Revista Brasileira de Terapia Intensiva. 2010 Oct/Dec;22(4):369-74.

6. Aurélio FS, Tochetto TM. Mensuração do ruído em uma Unidade de Terapia Intensiva Neonatal. Acta Pediátrica Portuguesa. 2010;41(2):64-8.

7. Cardoso MVLM, Chaves EMC, Bezerra MGA. Ruídos e barulhos na unidade neonatal. Rev Bras Enferm. Brasília [periódico internet] 2010 jul-ago; [acesso em $2011 \mathrm{dez}$ 16];63(4):561-6. Disponível em: http://www.scielo.br/pdf/reben/v63n4/10.pdf

8. Rodarte MDO, Scochi CGS, Leite AM, Fujinaga CL, Zamberlan NE, Castral TC. O ruído na manipulação das incubadoras: implicações para o cuidado de enfermagem. Rev. latino-am. enfermagem. 2005 Jan./fev;13(1):79-85.

9. Felli VEA, Tronchin DMR. A Qualidade de vida no trabalho e a saúde do trabalhador de enfermagem. In: Kurcgante P (Coord). Gerenciamento de Enfermagem. Rio de Janeiro: Guanabara Koogan; 2005.

10. Fress MFR. Avaliação dos níveis de ruído em estabelecimentos assistenciais de saúde: estudo de caso [dissertação]. Santa Maria(RS): Universidade de Federal de Santa Maria;2006 [acesso em 2011Jul 20]. Disponível em:

http://w3.ufsm.br/ppgec/wp-content/uploads/diss_ maria_de_fatima.pdf

11. Ichisato SMT, Scochi CGS. Ruídos na unidade de cuidado intensivo neonatal durante as passagens de plantão (enfermagem e/ou médica) e visita médica. Rev.Ciência, Cuidado e Saúde. 2006;5(supl):127-33.

12. Ferreira, Luiza Maria Bastos. Ruídos no Centro Cirúrgico: ecos do ambiente na saúde do trabalhador de Enfermagem [dissertação]. (Mestrado em Enfermagem) - Universidade do Estado do Rio de
Janeiro, Rio de Janeiro, 2003. Disponível em: http:// pesquisa.bvsalud.org/regional/resources/lil-398409 13. Curado JAF, Rabelo WCSV, Alves W, Perini RF, Siqueira PH. A incidência de PAIR na lavanderia de um hospital universitário. Arq. Otorrinolaringol. 2001 Abr/Jun; 5(2):113-6.

14. Muniz LMN, Stroppa MA. Desconfortos dos pacientes internados na UTI,quanto a poluição sonora. Revista de Administração Hospitalar e Inovação em Saúde. jul./dez. 2009 disponível em :http://www.face.ufmg.br/revista/index.php/rahis/ article/viewFile/808/682

15. Freitas SF, Clímaco RSC. Análise do conforto sonoro em hospitais de Brasília. V Encontro Nacional em Ambiente Construído, 1999.

16. Corrêa A L.Ruído: Níveis de pressão sonora captados no interior e exterior de incubadora em unidade de cuidados intensivos neonatal[dissertação]. São José dos Campos(SP):programa de pós-graduação em bioengenharia do Instituto de Pesquisa e Desenvolvimento da Universidade do Vale da Paraíba; 2005.disponível em: http://biblioteca. univap.br/dados/000001/00000111.pdf

17. ANVISA: Agência Nacional de Vigilância Sanitária. Segurança no ambiente hospitalar. [Manual na Internet]. [s.d.] [acesso em 2011 Maio 9]; [aproximadamente 172 p.]. Disponível em: http://www.anvisa.gov.br/servicosaude/manuais/ seguranca_hosp.pdf

18. Silva MC, Orlandi CG, Chang EM, Siviero J, Pinto MM, Armellini PFS et al. Níveis de ruído na lavanderia de um hospital público Rev. CEFAC. São Paulo disponível em: http://www.scielo.br/pdf/ rcefac/2010nahead/180-09.pdf

19. Miasso Al, Silva AEB de CS, Cassiani SH de BC, Grou CR, Oliveira RC de, Fakih FT. O processo de administração de medicamentos: identificação de problemas para propor melhorias e prevenir erros de medicação. Rev Latino-am Enfermagem. 2006 Maio/jun;14(3):354-63.

20. WHO: World Health Organization. Guidelines for Community Noise. Noise sources and their measurement. 1999. [acesso em: 2011 Ago 10]. Disponível em: http://www.who.int/docstore/peh/ noise/guidelines2.html

21. ABNT: Associação Brasileira de Normas Técnicas-NBR 10151. Avaliação do ruído em áreas habitadas visando o conforto acústico da comunidade. Rio de Janeiro; 2000.

22. ABNT: Associação Brasileira de Normas Técnicas-NBR 10152. Níveis de ruído para conforto acústico. Rio de Janeiro;1987.

23. Brasil. Ministério do Trabalho. Limite de tolerância. Portaria 3214 de 08 de junho de 1978 -NR 15 Anexos 1 e 2 [Norma regulamentadora 
na internet]; 1978 Jun [acesso em: 2011 Set. 28]:[aproximadamente 3 páginas]. Disponível em: $\quad$ http://portal.mte.gov.br/legislacao/normaregulamentadora-n-15.htm

24. Brasil. Manual de aplicação da norma regulamentadora n.0 17. Brasília: Ministério do Trabalho, 2002.

25. Macedo ISC, Mateus DC, Costa EMGC, Asprino ACL, Lourenço EA. Avaliação do ruído em Unidades de Terapia Intensiva. Braz. j. otorhinolaryngol. (Impr.); 2009 Nov./dez;75(6):844-6.

26. Oliveira EB, Lisboa MTL. The impact of noise for the nursing workers health and the work process. Online Brazilian of Nursing [periódico na internet] 2007;6(3):1237- 266. Available from:http:// www.uff.br/ objnursing/index.php/nursing/article/ view/j.1676- 4285.2007.1237/266.

27. Nogueira MFH, DI Piero KC, Ramos EG, Souza MN, Dutra MVP. Mensuração de ruído sonoro em unidades neonatais e incubadoras com recém-nascidos: revisão sistemática de literatura. Rev. latino-am. enfermagem [periódico na Internet]. 2011 Jan./fev. [acesso em: 2011 Maio 10];19(1):[aproximadamente 10 páginas]. Disponível em: http://www.scielo.br/pdf/rlae/v19n1/ pt_28.pdf.

28. Zamberlan NE, Ichisato SMT, Rodarte MDO, Fujinaga CI, Hass VJ, Scochi CG S. Ruído em uma Unidade de Cuidado Intermediário Neonatal de um
Hospital Universitário. Cuidado Ciência e Saúde. 2008 Out/Dez;4(7):431-8.

29. Baggio MCF, Marziale MHP. A participação da enfermeira do trabalho no programa de conservação auditiva. Rev Latino-am Enfermagem. 2001 Set./ out. 9(5):97-9.

30. Peduzzi M. Equipe multiprofissional de saúde: conceito e tipologia. Rev Saúde Pública [periódico na Internet]. $2001 \mathrm{Fev}$ [acesso em: $2011 \mathrm{Jul}$ 26];35(1): [aproximadamente 7 páginas]. Disponível em: <http://www.scielosp.org/scielo.php?script=sci arttext\&pid=S0034-89102001000100016\&Ing=en\& nrm=iso > access on 26 July 2011. doi: 10.1590/ S0034-89102001000100016.

31. Fortuna CM, Mishima SM, Matumoto S, Pereira MJB. O trabalho de equipe no Programa de Saúde da Família: reflexões a partir de conceitos do processo grupal e de grupos operativos. Rev. latino-am. Enfermagem. 2005 Mar./abr;13(2):262-8.

32. Falk AS, Woods NF. Hospital noise - levels and potential health hazards. N Engl J Med. 1973 Oct;289(15):774-81.

33. Hilton A. The hospital racket: how noisy is your unit? Am J Nurs.1987 Jan; 87(1):59-61.

34. Tamez RN, Silva MJP. Enfermagem na UTI neonatal: Assistência ao recém-nascido de alto risco. Rio de Janeiro: Guanabara Koogan, 2002.

35. Bistafa S. Acústica aplicada ao controle do ruído. 2 edição. São Paulo: Blucher, 2011.

http://dx.doi.org/10.1590/1982-021620140412

Recebido em: 17/01/2012

Aceito em: 09/08/2012

Endereço para correspondência:

Walderes Aparecida Filus

Rua Padre Agostinho, 2677

Curitiba - PR

CEP: 80710-000

E-mail: silus@terra.com.br 